\title{
Pleural tuberculosis
}

\author{
Ashley Liou, DOa (D), Carlos E. Rodriguez-Castro, MD ${ }^{\mathrm{a}}$ (D), Abel Rodriguez-Reyes, MD ${ }^{\mathrm{b}}$, Riyam Zreik, MD , \\ Shirley Jones, $\mathrm{MD}^{\mathrm{a}}$, and Whitney Prince, $\mathrm{MD}^{\mathrm{a}}$ \\ aDepartment of Pulmonary and Critical Care, Baylor Scott \& White Medical Center-Temple and Texas A\&M College of Medicine, Temple, \\ Texas; 'Department of Medicine, Hospital Juan Maria De Salvatierra, Universidad Autonoma de Mexico, La Paz, Baja California Sur, Mexico; \\ cDepartment of Pathology, Baylor Scott \& White Medical Center-Temple and Texas A\&M College of Medicine, Temple, Texas
}

\begin{abstract}
A 35-year-old woman with previously untreated latent tuberculosis was admitted to the hospital for management of a right-sided empyema. After a prolonged hospitalization and several interventions, including chest tubes, bronchoscopy with bronchoalveolar lavage, and a video-assisted thoracoscopic surgery, positive acid-fast bacilli cultures on the initial thoracentesis ultimately led to the diagnosis of pleural tuberculosis. This case highlights the importance of utilizing a combination of diagnostic tests to diagnose pleural tuberculosis, especially in the setting of a negative pleural adenosine deaminase level.
\end{abstract}

KEYWORDS Adenosine deaminase; pleural biopsy; pleural effusion; pleural tuberculosis

\section{CASE DESCRIPTION}

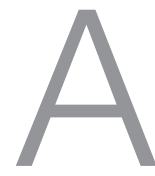

35-year-old woman who volunteered in a homeless shelter presented to the pulmonary clinic with a 3month history of worsening right-sided pleuritic chest pain associated with subjective fevers, chills, and night sweats. She had been diagnosed 10 months prior during an employee health screening with latent tuberculosis based on a purified protein derivative of $>27 \mathrm{~mm}$ of induration but did not undergo treatment. Physical exam was notable for a temperature of $37.7^{\circ} \mathrm{C}$, heart rate of 122 beats per minute, and absent lung sounds halfway up her right lower lung field. Her hemoglobin level was $10.4 \mathrm{~g} / \mathrm{dL}$, the platelet count was $521 \times 10^{9} / \mathrm{L}$, and human immunodeficiency virus serology was negative. A chest radiograph showed a large right pleural effusion, and a subsequent computed tomography scan of the chest revealed a loculated effusion without evidence of parenchymal disease (Figure 1). Initial thoracentesis showed turbid fluid with a $\mathrm{pH}$ of 7.31 ; red blood cell count, $820 / \mu \mathrm{L}$; white blood cell count, $920 / \mu \mathrm{L}$ with $97 \%$ lymphocytes; lactate dehydrogenase, $1010 \mathrm{IU} / \mathrm{L}$; glucose, $54 \mathrm{mg} / \mathrm{dL}$; total protein, $5.0 \mathrm{~g} / \mathrm{dL}$; and adenosine deaminase (ADA), <1.6 U/L. Complicated pleural effusion was diagnosed, and the patient was admitted to the hospital for further investigation.

A chest tube was placed, and the patient was started on antibiotics while waiting for cultures, fungal cultures, gram stain, and acid-fast bacilli analysis. Pleural fluid cultures grew coagulase-negative staphylococci, which was suspected to be a contaminant. Three acid-fast bacilli sputum stains were negative, and airborne precautions were discontinued. A subsequent bronchoscopy with bronchoalveolar lavage showed macrophages $91 \%$, lymphocytes $5 \%$, and neutrophils $4 \%$. Mycobacterium tuberculosis polymerase chain reaction tests from the fluid and bronchoalveolar lavage as well as acid-fast bacilli smears, fungal smears, and serology from the fluid and bronchoalveolar lavage were negative. The patient was given $5 \mathrm{mg}$ of intrapleural tissue plasminogen activator and $10 \mathrm{mg}$ of dornase alpha therapy, each in $50 \mathrm{~mL}$ of $0.9 \%$ sodium chloride, twice a day over 3 days. An additional chest tube was placed to assist with resolution of the empyema.

Due to the lack of improvement in symptoms and absence of a definitive diagnosis, video-assisted thoracoscopic surgery (VATS) was performed, which found a dense fibrinous exudate with pleural studding. Pathology reported numerous necrotizing granulomas (Figure 2). Following discharge, acid-fast bacilli pleural fluid cultures from the initial thoracentesis performed 3 weeks previously came back positive. $M$. tuberculosis was sensitive to rifampin, isoniazid, pyrazinamide, and ethambutol, and quadruple therapy was initiated. Additionally, acid-fast bacilli cultures from the VATS procedure came back positive after 4 weeks.

Corresponding author: Carlos E. Rodriguez-Castro, MD, Department of Pulmonary and Critical Care, Baylor Scott \& White Medical Center-Temple, 2401 S. 31st St., MS-09-C513-C, Temple, TX 76508 (e-mail: carlos.rodriguezcastro@bswhealth.org)

Color versions of one or more of the figures in the article can be found online at www.tandfonline.com/ubmc.

Received June 14, 2019; Revised July 8, 2019; Accepted July 18, 2019. 


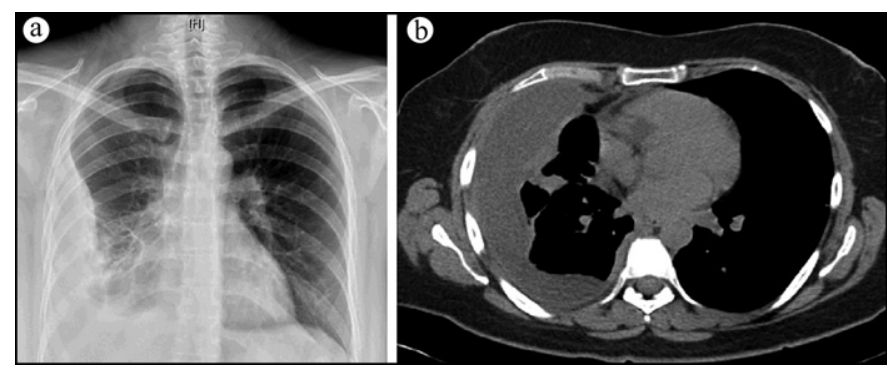

Figure 1. (a) Chest $x$-ray, anteroposterior view, demonstrating large, rightsided loculated effusion. (b) Sagittal section of CT chest similarly demonstrating right-sided loculated effusion without parenchymal disease.

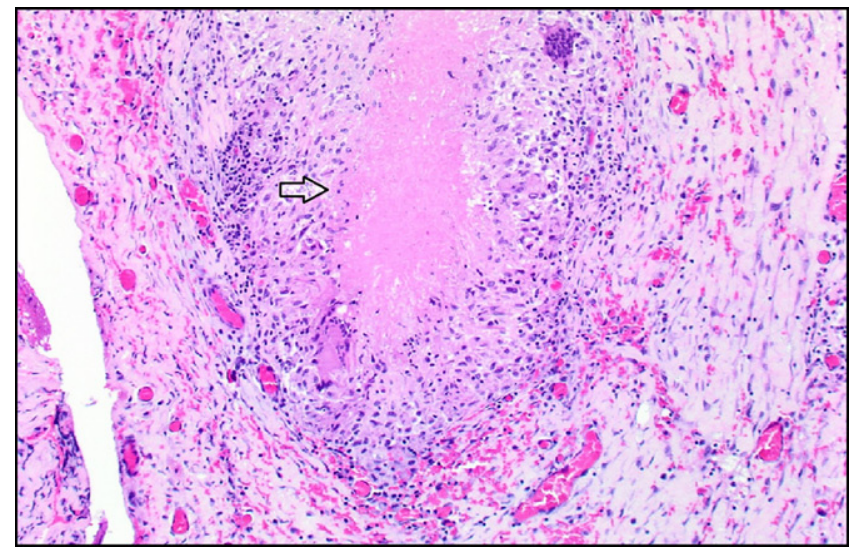

Figure 2. Necrotizing granulomas (arrow). Hematoxylin and eosin stain, original magnification $\times 400$.

\section{DISCUSSION}

In the USA, tuberculous pleurisy is fairly uncommon. Only 7549 cases of $M$. tuberculosis have been reported in the 10 years from 1993 to 2003, with 3.6\% accounting for all cases of pleural tuberculosis. ${ }^{1}$ The diagnosis of tuberculous pleurisy is usually made through a combination of clinical history, pleural fluid analysis (lymphocytic-predominant cell count, a protein concentration $>3.0 \mathrm{~g} / \mathrm{dL}$, elevated lactate dehydrogenase often $>500$ IU/L, and glucose level $<60 \mathrm{mg} / \mathrm{dL}$ ), positive cultures in the sputum or pleural fluid, and a positive ADA level $>40 \mathrm{U} / \mathrm{L} .{ }^{2} \mathrm{An}$ ADA level $>40 \mathrm{UI} / \mathrm{L}$ and a lymphocyte-to-neutrophil ratio $>0.75$ in the pleural fluid can be useful to establish a presumptive diagnosis of tuberculous pleural effusion, even when the acid-fast bacilli smear and culture are negative. ${ }^{3}$ Pleural effusions with an ADA level $<40 \mathrm{U} / \mathrm{L}$ are rarely caused by $M$. tuberculosis, denoting that the test has high negative predictive value and is an excellent test to rule out the infection. ${ }^{4}$

In our case, two acid-fast bacilli cultures from three different diagnostic methods came back positive, though timing from the thoracentesis allowed for earlier diagnosis. There were suggestions from the initial pleural fluid that pointed toward tuberculosis pleurisy. This included a predominant pleural fluid lymphocytosis and elevated lactate dehydrogenase. ${ }^{5}$ The distinctiveness of our case lies in a negative ADA and the sample used, because ultimately the pleural fluid was the first that grew acid-fast bacilli indicating the presence of M. tuberculosis. Not only is ADA a highly sensitive and specific test, but the negative predictive value of pleural ADA in a low-prevalence area for tuberculosis remains quite high. ${ }^{4,6}$ Because of delayed hypersensitivity in pleural tuberculosis, which occurs about $30 \%$ of the time, it can be difficult to isolate $M$. tuberculosis from pleural fluid samples. ${ }^{7}$ Samples obtained from bronchoscopy with bronchoalveolar lavage and VATS typically provide high and rapid diagnostic yield in undiagnosed exudative effusions, especially when there is associated parenchymal disease. ${ }^{5,8,9}$ Decortication also may have played a role in treatment, improving lung function and reducing residual pleural thickening. ${ }^{10}$

Early diagnosis and treatment of pleural tuberculosis reduces patient morbidity, prevents transmission, and reduces hospital stay. ${ }^{11}$ Thoracentesis, a modality often believed to be less sensitive than fiber-optic bronchoscopy with bronchoalveolar lavage and/or VATS, may play a larger role in establishing the diagnosis of pleural tuberculosis in combination with histology of the pleural biopsy.

\section{ORCID}

Ashley Liou (iD http://orcid.org/0000-0002-7716-5562

Carlos E. Rodriguez-Castro (D) http://orcid.org/0000-0003-2508-5787

1. Baumann MH, Nolan R, Petrini M, Lee YC, Light RW, Schneider E. Pleural tuberculosis in the United States: incidence and drug resistance. Chest. 2007;131:1125-1132. doi:10.1378/chest.06-2352.

2. Epstein DM, Kline LR, Albelda SM, Miller WT. Tuberculous pleural effusions. Chest. 1987;91:106-109. doi:10.1378/chest.91.1.106.

3. Lewinsohn DM, Leonard MK, LoBue PA, et al. Official American Thoracic Society/Infectious Diseases Society of America/Centers for Disease Control and Prevention clinical practice guidelines: diagnosis of tuberculosis in adults and children. Clin Infect Dis. 2017;64: e1-e33. doi:10.1093/cid/ciw694.

4. Blakiston M, Chiu W, Wong C, Morpeth S, Taylor S. Diagnostic performance of pleural fluid adenosine deaminase for tuberculous pleural effusion in a low-incidence setting. J Clin Microbiol. 2018;56: e00258-18. doi:10.1128/JCM.00258-18.

5. Ruan SY, Chuang YC, Wang JY, et al. Revisiting tuberculous pleurisy: pleural fluid characteristics and diagnostic yield of mycobacterial culture in an endemic area. Thorax. 2012;67:822-827. doi:10.1136/thoraxjnl-2011201363.

6. Jiménez Castro D, Díaz Nuevo G, Pérez-Rodríguez E, Light RW. Diagnostic value of adenosine deaminase in nontuberculous lymphocytic pleural effusions. Eur Respir J. 2003;21:220-224. doi:10.1183/ 09031936.03.00051603.

7. Porcel JM. Tuberculous pleural effusion. Lung. 2009;187:263-270. doi:10.1007/s00408-009-9165-3.

8. Baughman RP, Dohn MN, Loudon RG, Frame PT. Bronchoscopy with bronchoalveolar lavage in tuberculosis and fungal infections. Chest. 1991;99:92-97. doi:10.1378/chest.99.1.92.

9. Kirsch CM, Kroe DM, Azzi RL, Jensen WA, Kagawa FT, Wehner JH. The optimal number of pleural biopsy specimens for a diagnosis of tuberculous pleurisy. Chest. 1997;112:702-706. doi:10.1378/chest.112.3.702.

10. Vorster MJ, Allwood BW, Diacon AH, Koegelenberg CF. Tuberculous pleural effusions: advances and controversies. J Thorac Dis. 2015;7:981-991.

11. Lin MT, Wang JY, Yu CJ, Lee LN, Yang PC; TAMI Group. Mycobacterium tuberculosis and polymorphonuclear pleural effusion: incidence and clinical pointers. Respir Med. 2009;103:820-826. doi: 10.1016/j.rmed.2008.12.023. 